\title{
Quasilinear theory for relativistic particles
}

\author{
Zhong-Tian Wang ${ }^{1,2}$, Zhi-Xiong He ${ }^{1}$, Zhan-Hui $\mathrm{Wang}^{1}$, Min $\mathrm{Xu}^{1}$, Jia-Qi Dong ${ }^{1}, \mathrm{Na} \mathrm{Wu}^{2}$, \\ Shao-Yong Chen ${ }^{2}$, Chang-Jian Tang ${ }^{2}$ \\ ${ }^{1}$ Southwestern Institute of Physics, Chengdu, Sichuan, 610041, China \\ ${ }^{2}$ College of Physics Science and Technology, Sichuan University, Chengdu, Sichuan, 610065, China
}

\section{Email address:}

wangzt@swip.ac.cn (Wang Zhong-Tian)

\section{To cite this article:}

Zhong-Tian Wang, Zhi-Xiong He, Zhan-Hui Wang, Min Xu, Jia-Qi Dong, Na Wu, Shao-Yong Chen, Chang-Jian Tang. Quasilinear Theory for Relativistic Particles. American Journal of Modern Physics. Vol. 3, No. 5, 2014, pp. 207-210. doi: 10.11648/j.ajmp.20140305.13

\begin{abstract}
Quasilinear theory is developed by using canonical variables for relativistic particles. It is self-consistent, including momentum, pitch-angle, and spatial diffusions. By assuming the wave field is a superposition of known toroidal and poloidal Fourier modes, the quasilinear diffusion coefficients are written in a form which can be directly evaluated by using the output of a spectral full-wave solver of Maxwell equations in toroidal plasmas. The formalism is special for tokamaks which are axis-symmetric, therefore, it is simple and suitable for simulations of cyclotron heating, current drive and radio-frequency wave induced radial transport in ITER. PACS: 52.35.Py, 52.50.Sw, 52.35.Fa.
\end{abstract}

Keywords: Relativistic, Quasi-Linear, Three-Dimension Diffusion

\section{Introduction}

Interaction of radio-frequency $(\mathrm{RF})$ wave with plasma in magnetic confinement devices has been a very important discipline of plasma physics. The bounce-average is needed to approach more realistic description of wave-plasma interaction in a time scale longer than the kinetic time scales. The long-time evolution of the kinetic distribution can be treated with Fokker-Planck equation. The behavior of the plasma and the most interesting macroscopic effects are obtained by balancing the diffusion term with a collision term. For the relativistic particles the action and angle variables initiated by Kaufman [1] are introduced. The technique of the area-conserved transformation proposed by Lichtenberg and Lieberman [2] is employed. A new invariant which actually is an implicit Hamiltonian is formed by using bounce average and from which the bounce frequency and precession frequency can be calculated. Using new action and angle variables the relativistic quasi-linear equation is derived, including spatial diffusion. It is different from Brizard and Chan [3] where magnetic field does not have the toroidal component. An elegant form of the quasilinear kinetic equation including a term describing RF wave induced radial diffusion has been derived by Eriksson and Helander [4]. This paper is an extension of their work with relativistic effects. In most of the above derivations for the quasi-linear operator it is assumed that the wave field in the vicinity of the resonances can be represented in an Eikonal form. Here, the field is supposed as a superposition of given toroidal and poloidal Fourier modes, instead. This is the representation used to solve the Maxwell equation in tokamak plasmas with a spectral full-wave code, for example, TORIC [5]. For the circulating particles, under the conditions of small Larmor radius and first harmonic resonance, the diffusion coefficient is compatible with the numerical code developed by Cardinali et al. [6]. The rest of the paper is organized as follows. In next section the exact guiding center variables are derived with Hamiltonian transformation. The guiding center variables are derived with Hamiltonian transformation. The bounce averaged quasi-linear equation is carried out in Section 3. A brief summary is presented in the last section.

\section{Exact Guiding Center Variables}

In tokamak configuration, the relativistic Hamiltonian of a charged particle can be expressed as

$$
H=\sqrt{\left[\left(P_{R}-\frac{e}{c} A_{R}\right)^{2}+\left(P_{Z}-\frac{e}{c} A_{Z}\right)^{2}+\left(P_{\phi}-\frac{e}{c} R A_{\phi}\right)^{2} / R^{2}\right] c^{2}+m_{0}^{2} c^{4}}+e \Phi
$$

where AR, AZ, and A are the vector potential components of the magnetic field, the electrical potential which is 
assumed to be a function of poloidal magnetic flux, $\Psi, m_{0}$ is the rest mass, and e the charge. $P_{R}, P_{\phi}$ and $P_{z}$ are the canonical momenta conjugate to $\mathrm{R}, \phi$ and $\mathrm{Z}$ respectively,

$$
\begin{gathered}
P_{R}=m_{0} u_{R}+\frac{e}{c} A_{R} \\
P_{\phi}=R m_{0} u_{\phi}+\frac{\mathrm{e}}{\mathrm{c}} \mathrm{R} A_{\phi} \\
P_{Z}=m_{0} u_{Z}+\frac{e}{c} A_{Z}
\end{gathered}
$$

where $u=\gamma v$ and $\gamma=\left(1+u^{2} / c^{2}\right)^{1 / 2}$ is the relativistic factor.

The magnetic field can be expressed as

$$
B=\nabla \phi \times \nabla \Psi+I \nabla \phi
$$

where $\Psi$ is the poloidal magnetic flux, I is related to the poloidal current, $\mathrm{R}$ is the major radius. Then, in tokamaks, we have

$$
A_{R}=0, A_{Z}=-I \ln \frac{R}{R_{0}}, A_{\phi}=-\frac{\Psi}{R}
$$

We introduce a generating function for changing to the guiding center variables,

$$
F_{1}=-\frac{m_{0} \Omega_{0} R_{0}^{2}}{2} \exp \left(\frac{X}{m_{0} \Omega_{0} R_{0}}\right)\left(\ln \frac{R}{R_{0}}-\frac{X}{m_{0} \Omega_{0} R_{0}}\right)^{2} \operatorname{tg} \alpha-Z X
$$

where

$$
X=m_{0} \Omega_{0} R_{0} \ln \frac{R_{C}}{R_{0}}
$$

and $\Omega_{0}$ is the toroidal gyrofrequency taken absolute value for electron, $\quad \rho$ the Larmor radius, $\quad \alpha$ the gyrophase, and subscripts o and $\mathrm{c}$ refer to the values at the magnetic axis and the guiding center, respectively. $\mathrm{X}$ and $\alpha$ are the new coordinates conjugate to the momenta

$$
P_{X}=Z+\rho \sin \alpha+\frac{\rho^{2}}{4 R_{C}} \sin 2 \alpha
$$

and

$$
P_{\alpha}=\frac{1}{2} m_{0} \Omega_{C} \rho^{2}
$$

where $P_{X}$ is actually the guiding center of $\mathrm{Z}$ coordinate, $\mathrm{Zc}$. A momentum is turned to be a coordinate during area-conserved canonical transformation [3]. The other two canonical variables $P_{\phi}$ and $\phi$ do not change in the new coordinates. The old coordinates are connected with new ones through four identical equations:

$$
P_{R}=m_{0} \Omega_{c} \rho e^{\frac{\rho}{R_{c}} \cos \alpha} \sin \alpha
$$

$$
\begin{gathered}
P_{Z}=-X \\
R=R_{C} \exp \left(-\frac{\rho \cos \alpha}{R_{C}}\right)
\end{gathered}
$$

$$
Z=P_{X}-\rho \sin \alpha-\frac{\rho^{2}}{4 R_{C}} \sin 2 \alpha
$$

The Jacobian in the area-conserved transformation is unity [3], that is,

$$
d \tau=J d P_{\alpha} d P_{x} d P_{\phi} d \alpha d X d \phi
$$

and

$$
J=1
$$

The exact Hamiltonian for the relativistic particles is

$$
H=\sqrt{\left\{2 m \Omega_{c} P_{\alpha}\left[\left(\frac{R}{R}\right)^{2} \sin ^{2} \alpha+\cos \alpha\right]+\frac{1}{R^{2}}\left[P_{\phi}+e \Psi\right]^{2}\right\}^{2}+m_{b}^{2} c^{4}+e \Phi}
$$

It is suitable for particle simulation and to derive the equations of motion and Vlasov's equation.

\section{Quasi-Linear Equation}

For the gyro-kinetics, the Hamiltonian in Eq.(17) could be averaged as

$$
H=\sqrt{\left(2 m_{0} \Omega_{c} P_{\alpha}+m_{0}^{2} u_{\phi}^{2}\right) c^{2}+m_{0}^{2} c^{4}}+e \Phi .
$$

To derive the quasi-linear equation, we introcude a new invariant [3]

$$
\Pi=\frac{1}{2 \pi} \oint P_{x} d X
$$

For the trapped particles in the large aspect ratio configuration, $\varepsilon<<1$, we get

$$
\Pi_{t}=\frac{8 q R_{0} m_{0}\left(\varepsilon \Omega_{0} P_{\alpha} / m_{0}\right)^{0.5}}{\pi}\left[E\left(k_{1}\right)-\left(1-k_{1}^{2}\right) K\left(k_{1}\right)\right]
$$

which is the toroidal magnetic flux enclosed by drift surface. The bounce frequency and the procession frequency are obtained from Eq. (20) as in Ref. [7, 8],

$$
\omega_{b t}=\frac{\pi\left(\varepsilon \Omega_{0} P_{\alpha} / m_{0}\right)^{0.5}}{2 \gamma q R_{0} K\left(k_{1}\right)}
$$

and

$$
\omega_{\xi}=\frac{2 \Omega_{0} P_{\alpha}}{\gamma \Omega_{p} m_{0} R_{0}^{2}}\left[\frac{E\left(k_{1}\right)}{K\left(k_{1}\right)}-\frac{1}{2}\right]+\frac{4 \Omega_{0} P_{\alpha} \hat{s}}{\gamma \Omega_{p} m_{0} R_{0}^{2}}\left[\frac{E\left(k_{1}\right)}{K\left(k_{1}\right)}-\left(1-k_{1}^{2}\right)\right]
$$

where $\Omega_{p}$ is the poloidal gyro-frequency, $\varepsilon=r / R_{0}$, $k_{1}^{2}=\frac{u_{\phi 0}^{2}}{4 \varepsilon \Omega_{0} P_{\alpha}}$ and $\hat{s}$ is the magnetic shear. $E\left(k_{1}\right)$ and $K\left(k_{1}\right)$ are complete elliptic functions.

For the circulating particles, 


$$
\begin{gathered}
\Pi_{c}=\frac{\Omega_{0} m_{0} r^{2}}{2}+\frac{2 q R_{0} m_{0} u_{\phi 0} \sigma}{\pi} E(k) \\
\omega_{b c}=\frac{\pi u_{\phi 0} \sigma}{2 \gamma q R_{0} K(k)}
\end{gathered}
$$

and

$$
\omega_{\zeta c}=q \omega_{b c} \sigma+\frac{u_{\varphi 0}^{2}}{2 \gamma \Omega_{p} r R_{0}}\left[\frac{E(k)}{K(k)}-\left(1-\frac{k^{2}}{2}\right)\right]+\frac{u_{\varphi 0}^{2} \hat{s}}{\gamma \Omega_{p} R_{0}^{2}} \frac{E(k)}{K(k)}
$$

where $\sigma$ represents the direction of the circulating particle and $k^{2}=k_{1}^{-2}$. The bounce-averaged gyro-frequency for the trapped particles is

$$
\Omega=\frac{\Omega_{0}}{\gamma} *\left[1-2 \varepsilon\left(\frac{E\left(k_{1}\right)}{K\left(k_{1}\right)}-\frac{1}{2}\right)\right]
$$

while for the circulating particles, it is

$$
\begin{gathered}
\Omega=\frac{\Omega_{0}}{\gamma} *\left\{1-\frac{2 \varepsilon}{k^{2}(1+\varepsilon)}\left[\frac{E(k)}{K(k)}-\left(1-\frac{k^{2}}{2}\right)\right]\right\} \\
P_{\zeta}=-\frac{e}{c} \Psi_{0}=\Psi^{\bullet}
\end{gathered}
$$

New momenta $\Pi, P_{\alpha}$ and $P_{\zeta}$ are conjugate to $\eta, \alpha$ and $\zeta$, respectively, where $\Psi^{\bullet}$ is actually the position variable [9].

According to Liouville's theorem, the distribution function, $f$, satisfies Vlasov's equation:

$$
\frac{\partial f}{\partial t}+\dot{\eta} \frac{\partial f}{\partial \eta}+\dot{\xi} \frac{\partial f}{\partial \xi}+\dot{\alpha} \frac{\partial f}{\partial \alpha}+\dot{\Pi} \frac{\partial f}{\partial \Pi}+\dot{P}_{\xi} \frac{\partial f}{\partial P_{\xi}}+\dot{P}_{\alpha} \frac{\partial f}{\partial P_{\alpha}}=0
$$

where $f$ can be divided in two parts, the averaged part and oscillatory part,

$$
f=\bar{f}+\widetilde{f}
$$

The linear solution of Eq. (29) is

$$
\begin{aligned}
& \tilde{f}=-\left(n H_{1} \frac{\partial \bar{f}}{\partial P_{\xi}}+m H_{1} \frac{\partial \bar{f}}{\partial \Pi}+l H_{1} \frac{\partial \bar{f}}{\partial P_{\alpha}}\right) / \\
& \left(\omega-n \omega_{\xi}-m \omega_{b}-l \Omega\right)
\end{aligned}
$$

$\bar{f}$ is a function of $\Pi, P_{\alpha}, \Psi^{\bullet}$ and it satisfies the quasi-linear equation

$$
\frac{\partial \bar{f}}{\partial \tau}+L D L \bar{f}=C(\bar{f})
$$

where $\tau=t \nu, L=m \frac{\partial}{\partial \Pi}+n \frac{\partial}{\partial \Psi^{*}}+l \frac{\partial}{\partial P_{\alpha}}$ is differential operator of actions, $C$ is approximated by the linear truncated operator in the relativistic formulation [10] and normalized by $v$ which is the collision frequency, $D$ is the diffusion coefficient

$$
D=\pi H_{1}^{2} \delta\left(\omega-n \omega_{\xi}-m \omega_{b}-l \Omega\right) / v
$$

We set the normalized Hamiltonian $\hat{H}_{1}=\frac{H_{1}}{\Omega_{0} P_{\alpha 0}}$. All the frequencies are normalized by $\Omega_{0}$. All the momentums normalized by $P_{\alpha 0}$. Then we have

$$
\begin{gathered}
\frac{\partial \bar{f}}{\partial \tau}+\hat{L} \hat{D} \hat{L} \bar{f}=C(\bar{f}) \\
\hat{L}=m \frac{\partial}{\partial \hat{\Pi}}+n \frac{\partial}{\partial \hat{\Psi}^{\bullet}}+l \frac{\partial}{\partial \hat{P}_{\alpha}}
\end{gathered}
$$

and

$$
\hat{D}=\pi \hat{H}_{1}^{2} \delta\left(\hat{\omega}-n \hat{\omega}_{\xi}-m \hat{\omega}_{b}-l \hat{\Omega}\right) / \hat{v}
$$

The pitch angle variable is denoted in Ref. [11] as

$$
\lambda=\frac{P_{\alpha}}{H}
$$

We have $\lambda \Omega_{c}=\frac{u_{\perp}^{2}}{u^{2}}=\sin ^{2} \theta$, where $\theta$ is the pitch angle, $\frac{\partial}{\partial H}=-\frac{\lambda^{2} \partial}{P_{\alpha} \partial \lambda}=\frac{\left(1-\mu^{2}\right)^{2} \partial}{2 \Omega P_{\alpha} \mu \partial \mu} \quad, \quad \frac{\partial}{\partial \Pi}=\frac{\partial H}{\partial \Pi} \frac{\partial}{\partial H}=\omega_{b} \frac{\left(1-\mu^{2}\right)^{2}}{2 \Omega_{0} P_{\alpha}} \frac{\partial}{\mu \partial \mu}$ and $\frac{\partial}{\partial \hat{\Pi}}=P_{\alpha 0} \frac{\partial H}{\partial \Pi} \frac{\partial}{\partial H}=\hat{\omega}_{b} \frac{\left(1-\mu^{2}\right)^{2}}{2 \hat{P}_{\alpha}} \frac{\partial}{\mu \partial \mu}$ where $\mu=\cos \theta$, and

$k_{1}^{2}=\frac{u_{\varphi 0}^{2}}{4 \varepsilon \Omega_{0} P_{\alpha}}=\frac{H-\Omega_{0} p_{\alpha}}{2 \varepsilon \Omega_{0} P_{\alpha}}=\frac{1-\Omega_{0} \lambda}{2 \varepsilon \Omega_{0} \lambda}=\frac{\cos ^{2} \theta}{2 \varepsilon\left(1-\cos ^{2} \theta\right)}=\frac{\mu^{2}}{2 \varepsilon\left(1-\mu^{2}\right)}$

For one harmonic, it has

$H_{1 l}=\left[\left(e \Phi_{k}-\frac{e}{c} u_{\zeta} A_{\phi k}\right) J_{l}\left(k_{\perp} \rho\right)-\frac{e}{c} u_{\perp} A_{\not k} \frac{J_{l-1}+J_{l+1}}{2}-\frac{e}{c} u_{\perp} A_{R k} \frac{J_{l-1}-J_{l+1}}{2 i}\right]$.

$i^{l} \exp \left(i \vec{k} \cdot \vec{r}_{c}-i l \alpha_{0}\right) \exp (i n \zeta+i m \eta+l \alpha-i \omega)$

where $\vec{A}$ is vector potential of incident electromagnetic wave, $\vec{r}_{c}$ is the guiding center position, $\alpha_{0}$ is the angle between $\vec{k}$ and $-\mathrm{R}$ direction in the cylindrical coordinates. And it then gets

$$
H_{1 l} \cdot H_{1 l}^{*}=\left[\left(e \Phi_{k}-\frac{e}{c} u_{\xi} A_{\phi k}\right) J_{l}\left(k_{\perp} \rho\right)-\frac{e}{c} u_{\perp} A_{\not k k} \frac{J_{l-1}+J_{l+1}}{2}-\frac{e}{c} u_{\perp} A_{R k} \frac{J_{l-1}-J_{l+1}}{2 i}\right]^{2}(3
$$

where $H_{1 l}^{*}$ and $H_{1 l}$ are conjugate.

For the relativistic circulating particles, the resonant term in Eq.(36) is written as

$$
\delta\left(\hat{\omega}-n \hat{\omega}_{\xi}-m \hat{\omega}_{b}-l \hat{\Omega}\right)=\frac{c}{q R_{\theta} \omega \hat{b} \hat{\omega}} \delta\left(N_{r s}-N_{\|}\right)=\frac{2 K(k)}{\pi} \cdot \frac{\gamma c}{\hat{\omega} u_{\phi 0}} \delta\left(N_{r e s}-N_{\|}\right)
$$




$$
\hat{D}=\pi \hat{H}_{1}^{2} \delta\left(\hat{\omega}-n \hat{\omega}_{\xi}-m \hat{\omega}_{b}-l \hat{\Omega}\right) / \hat{v}=\pi \hat{H}_{1}^{2} \frac{2 K(k)}{\pi} \cdot \frac{\gamma c}{\hat{\omega} u_{\phi 0}} \delta\left(N_{r e s}-N_{\|}\right)
$$

where $\gamma$ is relativistic factor, $N_{\|}=\frac{c}{q R_{0} \omega}(q n+m)$ and $N_{\text {res }}=\frac{2 K(k)}{\pi} \cdot \frac{c}{u_{\phi 0}}\left(\gamma-\frac{\Omega_{0}}{\omega}\right)$. It is consistent with the code developed in Frascati by Cardinali [6] if $\varepsilon<<1$, that is, $\mathrm{k}$ approaches zero. The $\delta$ function can be expressed in following form,

$$
\delta\left(N_{r e s}-N_{/ /}\right)=\pi^{-0.5} \frac{1}{\Delta N_{/ /}} \exp \left[-\left(N_{r e s}-N_{/ /}\right)^{2} / \Delta N_{/ /}^{2}\right]
$$

Eqs. (34), (35) and (41) are the bounce averaged the quasi-linear equation for relativistic particles.

\section{Summary}

The action and angle variables initiated by Kaufman are used [1]. The area-conserved transformation is employed [2]. The bounce-averaged quasi-linear Fokker-Planck equation for the relativistic particles is rigorously derived, including momentum, pitch angle, and spatial three-dimensional diffusion. Equations (34), (35), and (36) are the main results of the work. The diffusion coefficient expressed in (36) is similar to the one obtained by Eriksson and Helander [4]. Most of the past derivations of the quasi-linear operator assume that the wave field in the vicinity of the resonances can be represented in an Eikonal form. Here, the field is supposed, instead, to be given as a superposition of toroidal and poloidal Fourier modes. This is the representation used to solve Maxwell equation in tokamak plasmas with spectral full-wave code, for example, TORIC [5]. The formalism is special for tokamaks which are axis-symmetric, so it is simple and suitable for simulation of cyclotron heating, current drive, and radiofrequency wave-induced radial transport in ITER.

\section{Acknowledgments}

The authors would like to give special thanks to Dr. A. Cardinali for his report and his code left here during his visit to SWIP. Z. T. Wang would like to thank Dr. R. D. Hazeltine and Dr. P. Morrision for the checking generating function and many helpful discussions when he worked at the Institute for Fusion Studies University of Texas at Austin. This work was supported by Natural Science Fund No. 11261140327, No.11005035, No. 11205053, No.11105046 and The National Magnetic Confinement Fusion Science Program (Grant No. 2013GB107002).

\section{References}

[1] Kaufman A N 1972 Phys. Fluids 151063

[2] Lichtenberg A J and Lieberman M A 1983 Applied Sciences 38 (Springer-Verlag New York Inc.)

[3] A. J. Brizard and A. A. Chan, "Relativistic quasilinear diffusion in axisymmetric magnetic geometry for arbitrary-frequency electromagnetic fluctuations," Physics of Plasmas 11, no. 9, pp. 4220-4229, 2004.

[4] L.-G. Eriksson and P. Helander, "Monte Carlo operators for orbit-averaged Fokker-Planck equations," Physics of Plasmas, vol. 1, no. 2, pp. 308-314, 1994.

[5] M. Brambila, Plasma Physics and Controlled Fusion, vol. 41, p. 1, 1999.

[6] A. Cardinali, L.Morini, and F. Zonca, in Proceedings of the Joint Varenna-Lausanne InternationalWorkshop on Theory of Fusion Plasmas, J. Conner, O. Sauter, and E. Sindoni, Eds., vol. 871, p. 292, American Institute of Physics, Varenna, Italy, 2006

[7] R. D. Hazeltine, S. M. Mahajan, and D. A.Hitchcock, "Quasilinear diffusion and radial transport in tokamaks," Physics of Fluids, vol. 24, no. 6, pp. 1164-1179, 1981.

[8] Z. T. Wang, Y. X. Long, J. Q. Dong, L. Wang, and F. Zonca, "Fishbone instability excited by barely trapped electrons," Chinese Physics Letters, vol. 23, no. 1, pp. 158-160, 2006.

[9] Hazeltine R D Mahajan S M and Hitchcock D A, Phys. Fluids, 24, 1164 (1972).

[10] B. Braams and C. F. F. Karney, Phys. Fluids B1, 1355(1989).

[11] R. D. Hazeltine and J. D. Meiss, Plasma Confinement Published bby Addison-Webley Publishing Company, (1992). 